\title{
Überflüssiger Vorschlag zu unnötiger Gesetzgebung
}

\section{Marion Schafroth}

Dr. med., Vorstandsmitglied EXIT

Im Artikel «Suizidhilfe aus ärztlicher Sicht - die vernachlässigte Fürsorge» [1] erläutert Prof. Borasio die empirischen Daten zu Tötung auf Verlangen und assistiertem Suizid für diejenigen Länder, die diese legalisiert haben. In Bezug auf die Schweiz legte er seine persönlichen Gedanken zur möglichen Entwicklung dar und formulierte Empfehlungen für eine nationale Gesetzgebung.

Zwar hat Borasio in seinem Herkunftsland Deutschland einen liberalen Ruf, weil er den assistierten Suizid nicht grundsätzlich verbieten möchte, doch in der wesentlich liberaleren Schweiz würde eine Gesetzgebung zur Suizidhilfe nach seiner Vorstellung die persönliche Freiheit des urteilsfähigen Menschen, Art und Zeitpunkt des eigenen Todes selbst zu bestimmen, gegenüber heute gravierend einschränken. Diese Tatsache fordert eine Replik geradezu heraus. Als FMH-Mitglied mit 10-jähriger Erfahrung als EXIT-Konsiliarärztin und zusätzlich 6-jähriger Tätigkeit im EXIT-Vorstand (mit Zuständigkeit für den Bereich Freitodbegleitung) verfüge ich über eine fundierte Einsicht in Thematik und Praxis des assistierten Suizids in der Schweiz.

Zunächst gilt es, eine krasse Fehlaussage von Prof. Borasio richtig zu stellen: Er behauptet, dass «in den letzten Jahren eine zunehmende Anzahl von Menschen ohne körperliche Erkrankung Suizidhilfe in der Schweiz in Anspruch genommen hat». Wahr ist: Die Anzahl Freitodbegleitungen bewegt sich bei psychisch Kranken über die Jahre hin stabil im einstelligen Zahlenbereich, obwohl sich die Mitgliederzahl verdoppelt hat (EXIT ist die einzige Organisation, die bei schwerst psychisch Erkrankten nach einem speziellen Abklärungsprozess Freitodbegleitungen durchführt). Und wahr ist: Alle Suizidhilfeorganisationen führen grundsätzlich keine Freitodbegleitungen bei gesunden Menschen durch, sondern nur dann, wenn eine schwere Krankheit, schweres Leiden und/oder eine unerträgliche Behinderung vorliegen. Garantiert wird dies einerseits durch interne Richtlinien der Sterbehilfeorganisationen, andererseits dadurch, dass Ärzte ein Sterbemittel nur dann verschreiben dürfen, wenn eine Indikation gegeben ist. Borasios Überlegungen zu einem Gesetzesvorschlag für die Regelung der Suizidhilfe umfassen folgende Hauptaussagen:
1. Der Staat habe seine Fürsorgepflicht mit einer nationalen Gesetzgebung wahrzunehmen. Prozedurale Regeln für die Suizidhilfe seien unabdingbar, damit nicht die Suizidhilfeorganisationen ihre Tätigkeit und deren durch das Schweizer Strafrecht (Art. 115 SGB) gesetzte Grenzen einseitig mit dem Argument der Selbstbestimmung ausweiten könnten (z.B. in Richtung Suizidhilfe für Hochbetagte).

2. Würde Suizidhilfe auf chronisch Kranke und Hochbetagte ausgeweitet werden ("wie von EXIT propagiert»), so könnten sich kranke und alte Menschen aufgrund immanenter ökonomischer Mechanismen zunehmend zu einem Suizid gedrängt fühlen und der Aspekt der Fürsorge könnte vernachlässigt werden.

3. Suizidhilfe dürfe daher erst nach erfolgter Beratung durch «qualifizierte Ärzte» erfolgen (er nennt: Geriater, Psychogeriater, Palliativmediziner, evtl. auch speziell ausgebildete Psychotherapeuten), und die Rolle der Ärzteschaft solle vor allem in der «Beratung über mögliche lebensorientierte Alternativen zum Suizid» bestehen.

4. Es sei zu verhindern, dass Suizidhilfeorganisationen von der Bevölkerung weiterhin als primäre Ansprechpartner bezüglich Suizidhilfe wahrgenommen werden, denn diese Organisationen könnten den Aspekt der Fürsorge nicht abdecken, weil sie dazu weder ein Mandat noch die Kompetenz hätten.

Prof. Borasios Hauptaussagen stelle ich eine entschieden andere Sicht der Dinge gegenüber:

Ad 1.

Sterbehilfeorganisationen tun schon längst genau das, was Prof. Borasio sich von einem Gesetz und speziell dafür qualifizierten Ärzten verspricht: Sie nehmen ihre Sorgfalts- und Fürsorgepflicht wahr, beraten umfassend und verhelfen Menschen dazu, informierte Entscheidungen zu treffen. Dabei ist bereits jetzt immer zusätzlich beratend zumindest derjenige Arzt (zu 40\% der Hausarzt, zu 60\% ein Konsiliararzt) involviert, der letztendlich das Rezept für das Sterbemittel ausstellt. Die Praxis zeigt: Sterbewillige brauchen einen starken Willen, sich gegen fürsorgliche, manchmal auch offen paternalistische Meinungen durchzusetzen, und niemand wird zum Suizid gedrängt. 


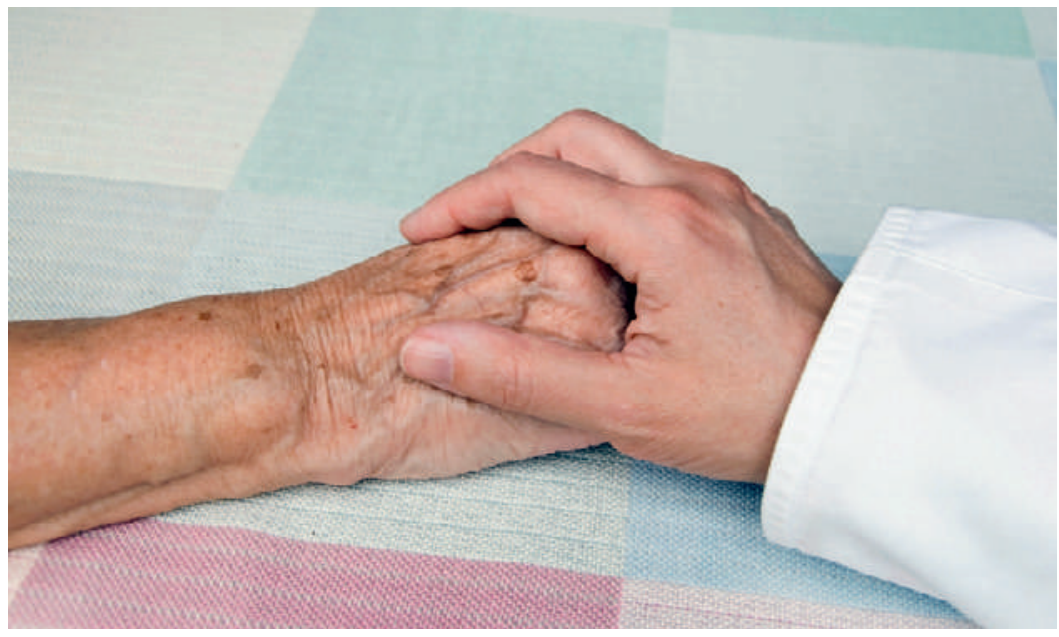

Ärzte begleiten ihre Patienten in allen Lebensphasen, das sollte bei Suizidhilfe nicht enden.

Ad 2.

Es gibt keine ethisch haltbare Begründung, warum Suizidhilfe nur bei einer terminalen Erkrankung zulässig sein soll. Unheilbares chronisches schweres Leiden und auch Alterspolymorbidität sind manchmal für einen Menschen sogar besonders leidstiftend und unerträglich, weil im Gegensatz zu einer terminalen Erkrankung keine Erlösung in Sicht ist. Ad 3.

Da ein atraumatischer Suizid nur mit Medikamenten durchführbar ist, die gemäss Gesundheitsrecht von einem Arzt verordnet sein müssen, ist die Rolle der Ärzteschaft gegeben. Ärzte haben das Recht, auf freiwilliger Basis das Sterbemedikament für einen wohlüberlegten Freitod zu verschreiben - eine Option, die über drei Viertel der Schweizer Bevölkerung (und Patientenschaft) als wünschbar erachten. Dazu braucht es keine besonders qualifizierten Spezialisten und keine neuen Regelungen. Sondern es braucht die vom Geist der Fürsorge getragene Bereitschaft der Ärzte, mit dem Patienten empathisch und ergebnisoffen sämtliche Optionen zu besprechen. Anstelle legislativer Tätigkeit durch die Politik erfordert dies eine Änderung der SAMW-Richtlinien in dem Sinne, dass Suizidhilfe generell als freiwillige ärztliche Tätigkeit gilt [2].

Ad 4.

Solange die SAMW-Richtlinien [3] und wesentliche Repräsentanten wie z.B. der Präsident der FMH daran festhalten, dass Suizidhilfe keine ärztliche Aufgabe sei, und Suizidhilfe auf das kurz bevorstehende Lebensende beschränken wollen [4], ist es für einen Menschen mit Sterbewunsch in vielen Fällen nur dank der Existenz von Sterbehilfeorganisationen möglich, in legalem Rahmen eine Freitodbegleitung zu erlangen. Denn diese Organisationen stellen nicht nur menschlich-einfühlsame Begleitung bei der Abklärung, Ausführung und behördlichen Nachuntersuchung eines Freitods zur Verfügung, sondern vermitteln nötigenfalls auch den Kontakt zu einem Arzt, der gewillt ist, der Eigenverantwortung des leidenden Patienten den nötigen Respekt zu zollen und nach erfolgter Überprüfung der Situation allenfalls das Rezept für das Sterbemedikament auszustellen. Die Sterbehilfeorganisationen arbeiten im Rahmen der geltenden Gesetze sorgfältig nach strengen Richtlinien und verfügen nach über 30-jähriger Existenz über viel Erfahrung. Im Mandat, Suizidwilligen zu helfen, ist die Verpflichtung zur Fürsorge aus dem Selbstverständnis der Sterbehilfeorganisationen gegeben, und die gut ausgebildeten Freitodbegleiterinnen und -begleiter verfügen über die Kompetenz und den Willen, die nötige Fürsorge zu leisten. Eine generell verbesserte Kooperation zwischen Ärzteschaft und Sterbehilfeorganisationen würde in dieser Hinsicht zusätzlich positive Wirkung entfalten.

Ärzte begleiten ihre Patienten in allen Lebensphasen, sei es präventiv, therapeutisch oder palliativ. Diese fürsorgliche Haltung sollte nicht ausgerechnet dann enden, wenn ein Patient den Wunsch nach Suizidhilfe äussert. Anstelle von Tabuisierung sind Aufklärung und Beratung zu allen bestehenden Alternativen gefragt. Beides, Palliative Care und assistierter Suizid, sind humane und mit ärztlichem Ethos vereinbare Wege, Leiden zu lindern oder zu beenden. Die in historischen Dimensionen betrachtet völlig neu aufgetretene Problemstellung, dass manche Menschen dank hochentwickelter Zivilisation und Medizin sehr alt, hochgebrechlich und abhängig und dabei am Nicht-sterben-Dürfen leidend werden, ist mit Jahrhunderte alten Handlungsanweisungen (wie dem Eid des Hippokrates, den in der Schweiz ohnehin kein Mediziner ablegt) und paternalistischer Gesetzgebung schlicht nicht zu lösen. Nicht nur Präventions- und Behandlungsmethoden haben mit der Zeit zu gehen, sondern auch die Rolle des Arztes am Lebensende. Suizidhilfe und Palliative Care sind wie Yin und Yang - entgegengesetzt und dennoch ergänzend aufeinander bezogen.

\section{Literatur}

1 Borasio GD. Suizidhilfe aus ärztlicher Sicht - die vernachlässigte Fürsorge. Schweiz Ärztezeitung. 2015;96(24):889-91.

2 Schafroth M. Suizidhilfe ist Teil der ärztlichen Aufgaben - die Zeit ist reif für eine Anpassung der SAMW-Richtlinien. Schweiz Ärztezeitung.2012;93(34):1226-7.

3 SAMW-Richtlinie «Betreuung von Patientinnen und Patienten am Lebensende». 2004 (aktualisiert 2012).

4 Tages-Anzeiger vom 8.8.2015: «Ärztepräsident will Diskussion über Sterbehilfegesetz".

Bildnachweis

() Totalpics | Dreamstime.com 